


\title{
Analyzing Disparities Trends for Health Care Insurance Coverage Among Non-Elderly Adults in the US: Evidence from the Behavioral Risk Factor Surveillance System, 1993-2009
}

\author{
Shireen Assaf, \\ University of Padua and ICF International \\ Stefano Campostrini \\ University of Venice at Ca' Foscari \\ Cinzia Di Novi \\ University of Venice at Ca' Foscari \\ Fang Xu \\ Northrop Grumman Information Systems \\ Carol Gotway Crawford, \\ Department of Biostatistics and Bioinformatics \\ Rollins School of Public Health \\ Emory University
}

\begin{abstract}
Access to health care in the United States remains greatly disproportionate across socioeconomic groups. It is not known, however, whether the disparities between the socioeconomic categories are increasing or decreasing. This analysis used a wellestablished non-parametric technique, employing time-varying coefficient models applied to data from the 1993 to 2009 US Behavioral Risk Factor Surveillance System (BRFSS). The analysis was able to show the changes in the odds ratios of having no health insurance plan for variables of interest over time, therefore highlighting the changes in the disparities between the categories of a variable over time. While other studies have attempted to show the changes in health insurance coverage by socioeconomic groups in different time periods, there is no study to date that has shown these changes as a smooth function with time, therefore providing a clearer picture of the changes in these disparities. The results of this analysis show, for instance, that when compared with individuals with a college education or greater, those with less than a high school education showed a steady increase in the odds ratios for having no health insurance. The same trend seems applicable although in a less-clear way to Hispanics and Non-Hispanic black race-ethnicities, compared with non-Hispanic whites (the reference race category). As measures of the Affordable Care Act are being gradually implemented, studies are needed to provide baseline information about health care access disparity, in order to gauge any changes in health care access over time; BRFSS can be a useful data source in accomplishing this task.
\end{abstract}

Keywords: USA, big data, disparities, health plan, health surveillance data, P-splines, temporal trends, varying coefficient model

JEL Codes: I14

Address for correspondence:

Shireen Assaf

ICF International,

530 Gaither Road, Suite 500,

Rockville, MD 20850, USA

e-mail: assaf@stat.unipd.it; shireen122@gmail.com

This Working Paper is published under the auspices of the Department of Economics of the Ca' Foscari University of Venice. Opinions expressed herein are those of the authors and not those of the Department. The Working Paper series is designed to divulge preliminary or incomplete work, circulated to favour discussion and comments. Citation of this paper should consider its provisional character.

The Working Paper Series is available only on line (http://www.unive.it/nqcontent.cfm?a id=86302) For editorial correspondence, please contact: wp.dse@unive.it
Department of Economics

Ca' Foscari University of Venice

Cannaregio 873, Fondamenta San Giobbe

30121 Venice Italy

Fax: ++390412349210 


\title{
Analyzing Disparities Trends for Health Care Insurance Coverage Among Non-Elderly Adults in the US: Evidence from the Behavioral Risk Factor Surveillance System, 1993-2009
}

\author{
Shireen Assaf, Stefano Campostrini, Cinzia Di Novi, \\ Fang Xu, Carol Gotway Crawford.
}

\begin{abstract}
Access to health care in the United States remains greatly disproportionate across socioeconomic groups. It is not known, however, whether the disparities between the socioeconomic categories are increasing or decreasing. This analysis used a well-established non-parametric technique, employing time-varying coefficient models applied to data from the 1993 to 2009 US Behavioral Risk Factor Surveillance System (BRFSS). The analysis was able to show the changes in the odds ratios of having no health insurance plan for variables of interest over time, therefore highlighting the changes in the disparities between the categories of a variable over time. While other studies have attempted to show the changes in health insurance coverage by socioeconomic groups in different time periods, there is no study to date that has shown these changes as a smooth function with time, therefore providing a clearer picture of the changes in these disparities.

The results of this analysis show, for instance, that when compared with individuals with a college education or greater, those with less than a high school education showed a steady increase in the odds ratios for having no health insurance. The same trend seems applicable-although in a less-clear way-to Hispanics and Non-Hispanic black race-ethnicities, compared with non-Hispanic whites (the reference race category). As measures of the Affordable Care Act are being gradually implemented, studies are needed to provide baseline information about health care access disparity, in order to gauge any changes in health care access over time; BRFSS can be a useful data source in accomplishing this task.
\end{abstract}

Keywords: U.S.A.; big data; disparities; health plan; health surveillance data; P-splines; temporal trends; varying coefficient model.

\section{Introduction}

Despite improvements in some health measures of Americans in the last decades and the increase in life expectancy (United Health Foundation, 2013; National Center for Health Statistics, 2013), disparities persist across certain groups -who continue to have poorer access to health care services as well as poorer health outcomes than their 
counterparts. Several factors have emerged and been identified in the literature as being key to being healthy and having access to health care. These factors include low socioeconomic status and lack of health insurance (William \& Collins, 1995; Williams \& Collins, 2001; Sudano \& Baker, 2006). The US stands alone among the industrialized countries in not providing health care coverage to all its citizens (Schoen et al., 2013). Despite the recent attempt of the Affordable Care Act (ACA) to expand health insurance, health care coverage and consequent access to health care services are still a problematic issue. The US National Health Interview Survey in the first three months of 2013 found 46 million (14.8

The aim of this study is to identify disparities in health insurance coverage for adults aged 18-64 years across different demographic and socioeconomic groups over time using U.S. BRFSS data from 1993-2009. The results, therefore, can be used as a baseline for future studies, particularly after regulations from the ACA, which was signed into law in March 2010, are in effect as this could have a direct effect on the levels of health insurance coverage in the different population subgroups. The interest here is not to study the trends in health care insurance coverage, but to study the how disparities are changing over time - an analysis, to our knowledge, that has yet to be done. In this study, we examined disparities by characteristics that included race, sex, household income, educational attainment, and age between 18 and 64 .

This study's analytical approach is new to BRFSS applications; it used time varying coefficient models that allow the coefficients of the variables in a regression model-for instance, a logistic regression as in those applied here-to vary over time, which therefore allows the study of the trends of the coefficients (readable as odds ratios in logistic regression models). This approach provides a method that treats time as a continuous variable while taking into consideration the relationship between the variables. Therefore, observing the trends in the odds ratio plots can highlight the trends in the disparities, since we are observing how the odds of a certain category of a variable is changing in terms of the reference category for that variable. This analysis is particularly interesting in this moment of change for the US, given the implementation in this year of the ACA. For this reason, before presenting our methods and analysis, the following section briefly discusses the past and present background of the US health care system.

\section{Institutional Background}

One way that the U.S. health care system is characterized-as with health systems in most countries- is by the ratio of private and public insurers funding the system. What is unique about the U.S. system is the dominance of the private element over 
the public one: coverage is provided mainly through private health insurance that is the largest component of the health care system. In the first three months of 2013, it was estimated that $23.9 \%$ of the U.S. population under the age of 65 was covered by a public health plan (Cohen \& Martinez, 2013). These programs include Medicare, Medicaid, the Children's Health Insurance Program (CHIP), state-sponsored or other government-sponsored health plans, and military plans (Cohen \& Martinez, 2013). This leaves $60.3 \%$ of those under the age of 65 covered by private health care plans, and $17.1 \%$ uninsured (Cohen \& Martinez, 2013).

The primary public US health coverage program is Medicare, a federal program enacted in 1965 and funded through social security payments. It provides health coverage mainly to people 65 years of age and older, some disabled people under 65 years of age, people with end-stage renal disease and Amyotrophic lateral sclerosis. Medicare has provided elderly Americans with basic health insurance coverage, with a number of gaps. Medicare does not cover the full range of health services needed by many elderly people: preventive care coverage is incomplete, and it lacks dental-, hearing-, and visionrelated benefits (AHRQ, 2007). In addition, Medicare does not cover chronic long-term care (LTC), most notably nursing home care for the disabled elderly (Rowland \& Lyons, 1996). In addition, over time other "holes" in coverage have been identified such as that of varying rates of coverage for prescription drugs (Medicare, 2014). To have additional coverage for these needs, most Medicare enrolees buy their own supplemental insurance coverage (i.e. Medigap insurance also known as Medicare supplement insurance).

Medicaid is funded jointly at the federal and state levels and is available for individuals of all ages and families with low income and limited resources who cannot afford proper medical care. Each state sets its own rules about eligibility and covered services. The eligibility depends on several factors such as age, pregnant status, disability, income and resources, as well as citizen/legal immigrant status. With the ACA, Medicaid has been expanded to include all nonelderly citizens and eligible legal residents whose family income does not exceed 133 percent of the Federal Poverty Line (FPL) (McDonough, 2014). Medicaid-ineligible people with incomes up to 400 percent of the poverty line can receive premium subsidies through tax credits for health plans offered through state health insurance exchanges.

Most non-elderly U.S. citizens obtain health insurance through their employers or organizations such as unions, professional associations, or other groups to which they belong; people who may not have access to group insurance(e.g. self-employed or people to whom employers do not offer health insurance plan) may choose to purchase their own individual health insurance directly from an insurance company (AHRQ, 2007). Individual insurance plans are traditionally much more expensive than group insurance since individuals will be responsible for paying the entire premium rather than sharing the cost with an employer and because of the type of the risk pool. Indeed, in the indi- 
vidual market individuals tend to be more heterogeneous in their risk level than those who have access to group insurance. Not surprisingly, they tend to face explicit variation in premiums that depends on characteristics thought to be predictive of expected benefits among which health risk is probably the most important. Approximately 5\% only purchase insurance on the private non group individual market. (AHRQ, 2007).

In the wake of the Great Recession in the late 2000s, the United States saw a crisis in the labor market with escalating unemployment that today stands at 6.7 percent (U.S. Bureau of Labor Statistics, 2014). Since employers provide health insurance as part of the benefits package for employees, the loss of a job has resulted in the loss of health benefits for millions of Americans, exposing individuals and families to potentially catastrophic health care costs in the event of a serious illness. Currently, workers who lose their job-based health benefits have few affordable insurance options. Unemployed individuals with incomes that are modest but too high to qualify for Medicaid, can buy health insurance through the individual insurance market but the majority of those who seek coverage in this market do not end up buying a plan because of the prohibitive cost. Under the COBRA (Consolidated Omnibus Budget Reconciliation Act), unemployed individuals, who are employed by a firm with 20 or more workers, have the right to temporary continuation of health coverage at group rates (for up to 18 months). Few people, however, decide to continue their coverage through COBRA since the participants are generally required to pay the entire premium themselves and plans tend to be too expensive. The new ACA legislation does not make any changes in COBRA; however, unemployed individuals may have expanded health insurance options, including subsidies to purchase insurance through exchanges, and expanded access to Medicaid coverage. At this time, however, roughly half of the states have indicated that they will implement the Medicaid expansion for 2014. In states that choose not to expand Medicaid, persons below $100 \%$ of FPL will not be eligible for either Medicaid or subsidies on the exchanges.

Even though losing or changing jobs may creates a gap in health insurance coverage between employed and unemployed individuals (Meyer et al., 2013), disparities in health care coverage also exist among other groups, especially younger and less educated individuals and racial/ethnic minority groups. In general, those with lower income, younger age, less education, and being of Hispanic ethnicity were found to have higher percentages of having no health insurance (Ahluwalia \& Bolen, 2008; Cohen \& Martinez, 2013; Strine et al., 2011). These disparities can also be quite large, for instance it was estimated from the U.S. National Health Interview Survey in 2013 that 42.6\% of 18-64 adults with no high school diploma were uninsured compared with $14.0 \%$ with more than a high school education, and an estimated $41.4 \%$ of uninsured Hispanics were found compared with 15.2\% uninsured non-Hispanic whites (Cohen \& Martinez, 2013). The same survey also found an estimated $39.1 \%$ of the poor-defined as those with an 
income below the poverty threshold given the family household size-were uninsured compared with $11.7 \%$ of the not-poor (defined as $200 \%$ above poverty threshold) in the 18-64 age category (Cohen \& Martinez, 2013). In addition, Ahluwalia \& Bolen (2008) have found an increase in uninsuredness for those with high school or less education, those aged 18-34, Hispanics, and the employed from 2001 to 2006, although the method used did not control for other variables and is not able to assess the gaps between the categories.

The ACA reform envisions important changes from 2014 for workers, younger adults, those on low income and those at high health risk. As far as access is concerned, in exchange for tax cuts, employers with over 50 employees are obliged to supply their employees insurance coverage, a provision enforced by a fine of 2,000 USD-per employee for each year of missed coverage. In view of this, therefore, there may be a real improvement in access to health care for many workers although this is not guaranteed as employers may find it more cost-effective to pay the fine rather than provide insurance coverage. Again concerning access an extension of the Medicaid program is envisaged from 2014 and changes in the implementation are happening, particularly where this, as mentioned bore, was left to state-level decisions. Moreover, the "Obamacare" reform will not allow insurance companies to fix premiums based on a patient's clinical history (i.e. pre-existing conditions). This substantially limits companies dumping or rather refusing to cover high-risk individuals through price policies (McDonough, 2014). Insurers will not be allowed to turn away people with pre-existing health conditions, cancel coverage when beneficiaries need expensive treatment, or charge women higher premiums than men. Beginning in 2014, all individuals will be required, with exceptions, to have health insurance or pay 695 USD per person, up to 2850 USD per family. Comprehensive coverage will be mandated, with caps on annual out-of- pocket costs.

In US, young adults have represented until today one of the largest segments of the U.S. population without health insurance. Thanks to Obama reform young adult children can stay on their parents' policies up to age 26 (an increase from age 18) (Connors \& Gostin, 2010; Light, 2011).Also college students who go to college fulltime are now able to be covered through their parents' insurance policies until age 26. Upon graduation, however, they lose their eligibility for family coverage. The current high unemployment rates across the country tend to exacerbate the difficulties young adults face in obtaining employment sponsored health insurance. Moreover, the number of standard full time permanent job in the last decade has decreased, while the non-standard work arrangements (temporary work, contingent, part-time contract etc.) have become much more common especially for young adults. Even when employed, young adults are typically employed through low-wage or temporary jobs that generally do not offer health insurance benefits (?Cohen \& Bloom, 2010). 


\section{Methods}

\subsection{Data}

BRFSS is the world largest telephone survey. It began collecting data from 15 U.S. states in 1984; by 1993 it had become a nationwide system with a total sample size that exceeded 100,000 a year (CDC, 2014; Mokdad, 2009). Sampling is conducting by taking a new random sample every month using random digit dialing from a land-line sampling frame. Since 2011, as an effort to increase response rates, cellular telephones were added to the sampling frame in the public use data. This addition was made after pilot surveys indicated that many population subgroups (particularly young adult members of these groups) were using cellular telephones only. This analysis does not use data after 2010, as the estimates between these two different sampling frames may not be directly comparable due the new weighting methodology used since 2011 (CDC, 2012). In addition, the US territories were not included in the analysis, as these data collection sites had very few observations, particularly in the earlier years of the survey.

Data from the US BRFSS from 1993 to 2009 were combined for performing the analysis. The data from 2010, 2008, and 2006 were excluded because participants were not asked questions on high blood pressure and high cholesterol status during these surveys, and these questions were required for this analysis. This study, therefore, is based on 15 years of data. The outcome variable is having no health insurance plan, which is taken from a question on whether the respondent currently has a health care plan. Seven independent variables were also constructed from the data, mainly to represent socio-demographic variables. These were age, sex, race, income, education level, work status, and number of health conditions. Only those aged 18-64 were included in the analysis; individuals aged 65 and over were excluded, as most members of this age group have health coverage through Medicare. The categories for the age variable, therefore, were those 18-34, 35-49, and 50-64 years of age. Race categories included non-Hispanic white, non-Hispanic black, Hispanic, and "other" race category, which includes all non-Hispanic minorities. Education was divided into four categories: less than grade 11 education level, high school diploma, some college, and more than college education. Work status contained three categories of working, not working, and student.

All the variables except for income and number of health conditions were taken directly from one question in the BRFSS dataset. The income variable was constructed by considering BRFSS household information on earnings and number of household members, as well as the poverty threshold for each year taken from the Department of Health and Human Services (US Department of Health and Human Services, 2013). Survey participants found to be earning less than the poverty threshold-for that year 
and number of household members-were considered to be in the low-income category. Those earning three times more than the poverty threshold were categorized as high income, and the remaining participants earning amounts between these two categories were placed in the median income category. Responses to the household earnings question often were missing (approximately 14\%), and therefore, after constructing the income variable using this question with the available data, the missing values were imputed from an ordered logistic regression model with income as the outcome variable and sex, age, race, education level and work status as the independent variables. The final sample size after imputing the missing values for income is 1,327,808 observations.

The number of health conditions variable was constructed by adding the responses to three questions in the BRFSS data set that asked participants if a doctor had ever told them that they have diabetes or high blood pressure or high cholesterol. Combining the responses from these questions resulted in four categories, which represent having no, one, two or three health conditions.

\subsection{Statistical Analysis}

Descriptive analysis was conducted to show distribution presented as proportions across various subgroups for not having a health care plan. Chi square tests were used to compare across groups. Multivariable logistic regressions were built to estimate odds ratios for each category of the demographic variables compared with the corresponding reference categories. A varying coefficient model was then constructed in order to observe how the coefficients (or odds ratios) were changing over time in order to study the trends of these disparities. Although BRFSS is a complex survey data, this analysis did not use weights to adjust for this, since the varying coefficient model contains the independent variables used in creating the weights, and therefore, are controlled for and should not affect the estimates. Descriptive statistics presented in Table 1 are consistent with this choice and consequently should be taken as references to better understand the models here proposed and not as unbiased estimates of the variable there reported.

This study's varying coefficient method requires a system that is continuously collecting health data, such as the US BRFSS, which has been collecting monthly data in several states since 1984 and nationwide since 1993 (Mokdad, 2009). The data are collected by taking a new random sample each month for a telephone interview; therefore, the data are not longitudinal. An advantage of this data source is that most of the questions required for this analysis are not changing significantly; therefore, a continuous data set can be created by combining data from several years of collection. The varying coefficient model approach also does not require aggregation of data such as in time series analysis methods, so that the information in the variation between the observation is not lost. These models were first discussed by Hastie \& Tibshirani (1993) 
and the description and estimation are well established in the literature. Their application to health surveillance data for studying trends, however, has not been performed. The analysis of trends using time varying coefficients has been discussed mainly for use with longitudinal data, in which the same observation is followed with time (Huang et al., 2004, 2002; Hoover et al., 1998).

The varying coefficient model is a direct extension of the generalized linear model. In a generalized linear model with a response variable $Y$ having a distribution from an exponential family, and independent variables $X_{1}, \ldots, X_{p}$, we have a model

$$
\eta=a_{0}+X_{1} a_{1}+\ldots+X_{p} a_{p}
$$

with the canonical link $\eta$ and link function $g()$. The coefficients $a_{1}, \ldots, a_{p}$ are constant. However, an example of a varying coefficient model can be written as

$$
\eta=a_{0}+X_{1} a_{1}\left(U_{1}\right)+\ldots+X_{p} a_{p}\left(U_{p}\right)
$$

with new variables $U_{1}, \ldots, U_{p}$, which are referred to as the effect modifiers Hastie \& Tibshirani (1993). Therefore, while in a generalized linear model, the coefficients are constant; in a varying coefficient model, we have coefficients that are a function of another variable (the effect modifier). The effect modifier can also be one single variable $U$ such as time, in the case of time varying coefficients. This case was used in this analysis, as we are interested in one effect modifier of time that is a variable constructed from the month and year of the interview for each observation. This statistical approach is different from the effect modifier used for constructing a time varying coefficient model in longitudinal studies, which usually use the age of the respondent to represent the effect modifier of time. The BRFSS is not a longitudinal study; the observations are not followed over time, and a new random sample is taken each month.

To construct the varying coefficient model, it is necessary to test whether these coefficients are constant or actually varying with time, which involves following two main steps: The first step is to fit a varying coefficient model for each independent variable while leaving all other independent variables with constant coefficients. This model is then tested against the null hypothesis, which contains the model where all the variables have constant coefficients, i.e.,

$$
\begin{aligned}
\mathbf{H}_{\mathbf{0}}: \operatorname{logit}(\text { nohplan }) & =\sum_{j=1}^{p} b_{j} Z_{j}, \\
\text { and } & \\
\mathbf{H}_{\mathbf{1}}: \operatorname{logit}(\text { nohplan }) & =\sum_{j=1}^{p} b_{j} Z_{j}+a_{1}(t) X_{1},
\end{aligned}
$$


nohplan is the outcome variable for having no health plan, $Z_{j}$ are the variables with constant coefficients $b_{j}$, and $a_{1}(t)$ is the time varying coefficient for the variable $X_{1}$. Using a $\chi^{2}$ of these two nested models would then show whether the time varying coefficient $a_{1}(t)$ is actually varying or should remain constant. This test is performed for each independent variable used in the analysis. The second step involves the building of the model by testing if the varying coefficients for a certain variable should remain in the model when other varying coefficients for another variable are already present, i.e.

$$
\begin{aligned}
& \mathbf{H}_{\mathbf{0}}: \operatorname{logit}(\text { nohplan })=\sum_{j=1}^{p} b_{j} Z_{j}+a_{1}(t) X_{1}, \\
& \mathbf{H}_{\mathbf{1}}: \operatorname{logit}(\text { nohplan })=\sum_{j=1}^{p} b_{j} Z_{j}+a_{1}(t) X_{1}+a_{2}(t) X_{2} .
\end{aligned}
$$

The null hypothesis includes the time varying coefficient $a_{1}(t)$, which was found to be significant in step one. The alternative hypothesis is now testing whether to include the time varying coefficient $a_{2}(t)$ for another variable $X_{2}$. These tests are then performed in a stepwise manner for all the variables in the analysis until the final model is reached.

The method described above involves using non-parametric techniques using splines (special piecewise polynomials) to fit the models. Using parametric techniques, while simpler, are not favored as the strong assumptions they require lack flexibility and could create misspecification of the data and large bias (Hastie \& Tibshirani, 1993; Fan \& Zhang, 2008). There are several non-parametric techniques that could be used, including smoothing spline, polynomial spline, and local regression. The present analysis using a type of penalized spline estimation method, P-spline estimation, which does not require selection of knots (the specific position points required to define the spline) and has a type of penalty that is less computationally expensive compared with the alternative smoothing spline penalty (Eilers \& Marx, 1996; Marx, 2010; Eilers \& Marx, 2002).

Computationally, the models were fit using P-spline estimation methods with a third degree B-spline and a second order difference penalty, as recommended by Eilers \& Marx (2002). A total of 45 knots were used (three for each year for the 15 years of observation). The models were fit using the $\mathrm{R}$ software program version 3.0.1 with the mgcv package and the bam function, which is designed for fitting models with big data (Wood, 2006). When the final time varying coefficient model is found, odds ratio plots could then be produced to easily interpret the results and trends. These odds ratio plots show the trends of the odds ratios for each population subgroup. 


\section{Results}

The disparities in terms of access to a health care plan can first be seen from the proportions as described in Table 1, where we can observe the distribution of the categories in the population for each variable as well as the distribution of these categories for those who have no health plan. Perhaps the most apparent differences in Table 1 are for the education and income variables. For instance while those with less than grade 11 education account for $6.8 \%$ of the population, $16.5 \%$ of those with no health insurance plan fall in this category. Similarly, while an estimated $6.5 \%$ of the population is in the low-income category, $18.3 \%$ of those with no health insurance are in this category. Disparities can also be seen between the race, age, and work status categories, but less so for the participants' sex and number of health conditions categories. A chi-squared contingency table test for each of the variables gave a significant p-value, which was expected with such high sample size, and indicates, therefore, that all the observed differences are also statistically significant.

Following the steps required for building of the time varying coefficient model resulted in a model in which all the independent variables had significant time varying coefficients. This final model can be written as:

$$
\begin{aligned}
\operatorname{logit}(\text { nohplan })=\sum_{j=1}^{p} b_{j} Z_{j} & +a_{0}(t)+a_{1}(t) \text { age }+a_{2}(t) \text { sex }+a_{3}(t) e d u+a_{4}(t) \text { work } \\
& +a_{5}(t) \text { race }+a_{6}(t) \text { income }+a_{7}(t) \text { healthcond }
\end{aligned}
$$

where the variable abbreviations are found in Table 1, logit (nohplan) is the logit of the binary outcome variable for having no health care plan; $Z_{j}$ are the covariates with constant coefficients $b_{j}$, and the time varying coefficients $\sum_{i=1}^{7} a_{i}(t)$ were found for all the independent variables used in the model. The coefficient $a_{0}(t)$ is the time varying intercept, which was also tested for inclusion in the model. Table 2 summarizes the estimates of this model with a parametric component and the non-parametric component represented by the splines of each category with time. Looking to the parametric component, we can observe the odds ratio (OR) with the $95 \%$ confidence intervals in Table 2. In general, younger age, male gender, lower education, not working, non-white race, and having lower income increase the odds of having no health plan. The greatest disparities were found in the income and education variables. For example, those with low income and median income have 6.76 (5.63-8.11) and 3.11 (2.65-3.66) times higher odds of having no health plan compared with those earning a high income. For education, respondents with less than a grade 11 education level have 2.77 (2.40-3.19) higher odds of having no health plan compared with those who have a university degree or more. The number of health conditions did not affect the outcome. 
Table 1: Descriptives of variables and proportion of non-elderly adults having no health insurance by socio-demographic variables and number of health conditions in the U.S. from 1993-2009. Sample size 1,327,808.

\begin{tabular}{|c|c|c|c|c|}
\hline \multicolumn{2}{|l|}{$\begin{array}{l}\text { Variable } \\
\text { (abbreviation) }\end{array}$} & $\begin{array}{r}\text { Total } \\
\%\end{array}$ & $\begin{array}{r}\text { No health } \\
\text { plan } \%\end{array}$ & $\begin{array}{c}\chi^{2} \\
\text { test }^{*}\end{array}$ \\
\hline \multicolumn{2}{|c|}{ Health care access } & - & 11.1 & \\
\hline \multirow{3}{*}{$\begin{array}{l}\text { Age } \\
\text { (age) }\end{array}$} & $18-34$ & 19.0 & 25.7 & $<0.001$ \\
\hline & $35-49$ & 39.0 & 36.7 & \\
\hline & $50-64$ & 42.0 & 37.6 & \\
\hline \multirow{6}{*}{$\begin{array}{l}\text { Sex } \\
(\text { sex }) \\
\text { Education } \\
(\text { edu) }\end{array}$} & Female & 60.8 & 61.0 & 0.002 \\
\hline & Male & 39.2 & 39.0 & \\
\hline & University+ & 37.2 & 17.8 & $<0.001$ \\
\hline & Some university & 28.4 & 28.5 & \\
\hline & High school & 27.6 & 37.2 & \\
\hline & $<$ grade 11 & 6.8 & 16.5 & \\
\hline \multirow{3}{*}{$\begin{array}{l}\text { Income } \\
\text { (income) }\end{array}$} & High & 39.8 & 16.3 & $<0.001$ \\
\hline & Median & 53.7 & 65.4 & \\
\hline & Low & 6.5 & 18.3 & \\
\hline \multirow{3}{*}{$\begin{array}{l}\text { Work status } \\
\text { (work) }\end{array}$} & Works & 72.3 & 59.8 & $<0.001$ \\
\hline & Student & 2.1 & 3.4 & \\
\hline & Does not work & 25.6 & 36.8 & \\
\hline \multirow{4}{*}{$\begin{array}{l}\text { Race } \\
\text { (race) }\end{array}$} & White & 79.4 & 67.6 & $<0.001$ \\
\hline & Black & 8.7 & 13.1 & \\
\hline & Hispanic & 6.7 & 12.3 & \\
\hline & Other & 5.3 & 6.9 & \\
\hline \multirow{4}{*}{$\begin{array}{l}\text { No. of health } \\
\text { conditions } \\
\text { (healthcond) }\end{array}$} & None & 52.8 & 54.1 & $<0.001$ \\
\hline & One & 30.4 & 28.1 & \\
\hline & Two & 13.6 & 14.2 & \\
\hline & Three & 3.2 & 3.7 & \\
\hline
\end{tabular}

Table 2 also provides the p-values of the spline estimates, which can provide an initial idea of whether the coefficients are significantly varying over time. The table shows that almost all the coefficients are significantly time varying except for age 5064, male, student, white race, and having three health conditions. These p-values can be underestimated (Wood, 2006), however, so p-value interpretation should be done with caution-especially if they are borderline significant. Therefore, although the only borderline significant p-value was for the black race category, to observe trends, it is better to rely on the reading and interpretation of the plots. 
Table 2: Summary of the estimates for the final time varying coefficient model for having no health care plan in the U.S. from 1993-2009.

\begin{tabular}{|c|c|c|c|}
\hline Variable & & OR (95\% C.I.) & p-value \\
\hline Age & $18-34$ & $1.87(1.70-2.07)$ & $<0.001$ \\
\hline \multirow[t]{4}{*}{ (Reference: 50-64) } & $35-49$ & $1.34(1.23-1.47)$ & $<0.001$ \\
\hline & $\mathrm{s}($ time$): 18-34$ & - & $<0.001$ \\
\hline & $\mathrm{s}($ time $): 35-49$ & - & $<0.001$ \\
\hline & $\mathrm{s}($ time $): 50-64$ & - & 0.518 \\
\hline Sex & Male & $1.32(1.19-1.46)$ & $<0.001$ \\
\hline \multirow[t]{2}{*}{ (Reference: Female) } & s(time):male & - & 0.997 \\
\hline & $\mathrm{s}($ time):female & - & $<0.001$ \\
\hline \multirow{7}{*}{$\begin{array}{l}\text { Education } \\
\text { (Reference: University or more) }\end{array}$} & Some university & $1.58(1.41-1.78)$ & $<0.001$ \\
\hline & High school & $1.64(1.47-1.83)$ & $<0.001$ \\
\hline & Less grade 11 & $2.77(2.40-3.19)$ & $<0.001$ \\
\hline & s(time):university or more & - & 0.002 \\
\hline & s(time):some university & - & 0.004 \\
\hline & s(time):high school & - & $<0.001$ \\
\hline & s(time):less grade 11 & - & $<0.001$ \\
\hline \multirow{5}{*}{$\begin{array}{l}\text { Work status } \\
\text { (Reference: Working) }\end{array}$} & Student & $1.08(1.00-1.15)$ & 0.094 \\
\hline & Does not work & $1.84(1.63-2.08)$ & $<0.001$ \\
\hline & s(time):working & - & $<0.001$ \\
\hline & $\mathrm{s}($ time$):$ student & - & 0.984 \\
\hline & $\mathrm{s}$ (time):does not work & - & $<0.001$ \\
\hline \multirow{7}{*}{$\begin{array}{l}\text { Race } \\
\text { (Reference: White) }\end{array}$} & Other & $1.08(0.92-1.27)$ & 0.456 \\
\hline & Hispanic & $1.76(1.09-2.84)$ & 0.054 \\
\hline & Black & $1.16(1.03-1.30)$ & 0.034 \\
\hline & s(time): white & - & 0.510 \\
\hline & s(time):other & - & 0.010 \\
\hline & s(time):hispanic & - & $<0.001$ \\
\hline & s(time):black & - & 0.024 \\
\hline \multirow{5}{*}{$\begin{array}{l}\text { Income } \\
\text { (Reference: High) }\end{array}$} & Median & $3.11(2.65-3.66)$ & $<0.001$ \\
\hline & Low & $6.76(5.63-8.11)$ & $<0.001$ \\
\hline & s(time):high & - & $<0.001$ \\
\hline & $\mathrm{s}($ time$):$ median & - & $<0.001$ \\
\hline & $\mathrm{s}($ time$):$ low & - & 0.015 \\
\hline \multirow{7}{*}{$\begin{array}{l}\text { Number of Health Conditions } \\
\text { (Reference: None) }\end{array}$} & One health condition & $1.01(0.95-1.08)$ & 0.715 \\
\hline & Two health conditions & $1.06(0.99-1.14)$ & 0.195 \\
\hline & Three health conditions & $1.04(0.93-1.16)$ & 0.601 \\
\hline & s(time):none & - & $<0.001$ \\
\hline & $\mathrm{s}($ time $):$ one & - & $<0.001$ \\
\hline & s(time):two & - & 0.005 \\
\hline & $\mathrm{s}($ time $):$ three & - & 0.983 \\
\hline
\end{tabular}


The odds ratio plots in the figures below show the change in the odds ratios over time for each category of a certain variable compared with the reference category of that variable. These plots clearly show which odds ratios are changing over time and which are constant. In general, we observed that all the odds ratio plots are exhibiting a non-constant trend over time, except for the male and student categories, which have constant odds ratios over time. Many of the plots suggest an improving situation in which there is a closing of the disparities for having a health care plan. This possibility can be seen for the age categories, the income levels, and the does-not-work category, in which we saw a decreasing odds ratio trend, compared with their respective reference categories (i.e. the groups are becoming more similar over time). Trends are positive, but gaps still remain: while the low-income category shows a decreasing odds ratio trend, additional analysis is needed, as this disparity remained quite large by the end of 2009 (OR of approximately 6, compared with that of the high-income category).

For the other variables, education and race, the disparities appear even to be increasing. For education we observe that odds ratio trends are increasing for all the education categories compared to the reference (university graduate or more category). For race, the odds ratio trends seem to be increasing at a slower rate. For the other race category (which includes non-Hispanic minority groups), the odds ratio began from below one (i.e. higher odds of having a health plan compared with white race), and increased to an odds ratio above one before plateauing approximately after the year 2000. For the number of health conditions, the trends are even more subtle. For those having both one or two health conditions, the odds ratio began from below one (i.e. higher odds of having a health plan compared with those with no health conditions), and increased to slightly above one or almost no difference between having or not having a health condition. There was a constant odds ratio trend for those having three or more health conditions.

\section{Discussion}

The results clearly show that great disparities existed in health care coverage, and that these disparities have increased over time for certain socioeconomic groups. The driving factors of the increased disparities seem to be related to education and race. For income level, while the disparities are quite large, the trends do appear to be decreasing over time. Ahluwalia \& Bolen (2008) have also shown an increase in uninsuredness for certain socio-demographic groups from 2001 to 2006, for the less educated, and among Hispanic groups, as was found in this analysis.

While income is the indicator that most directly measures material resources, education is perhaps the most basic socioeconomic component: lower education level may 


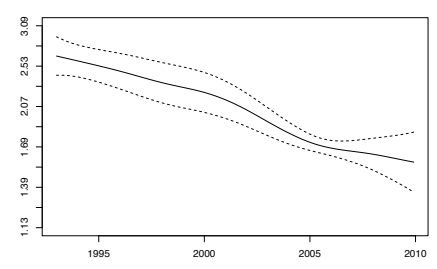

(a) age 18-34

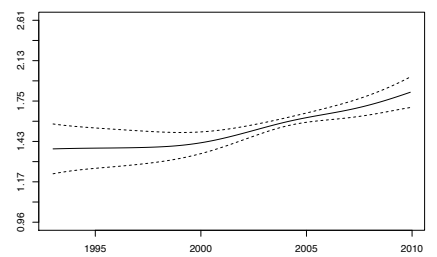

(d) some college

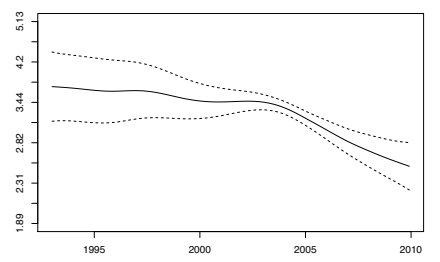

(g) median income

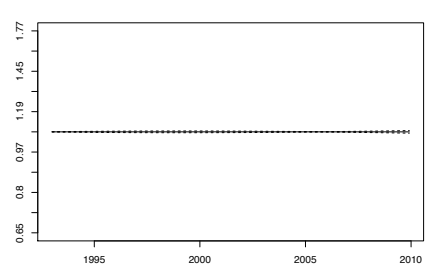

(i) student

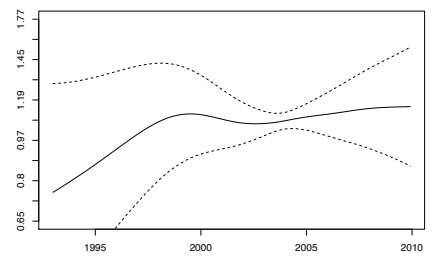

(k) other

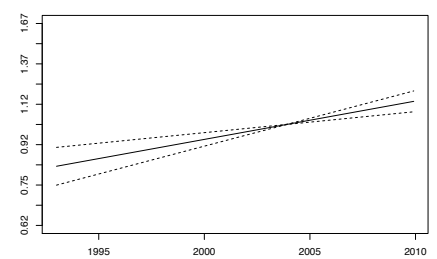

(n) one health condition

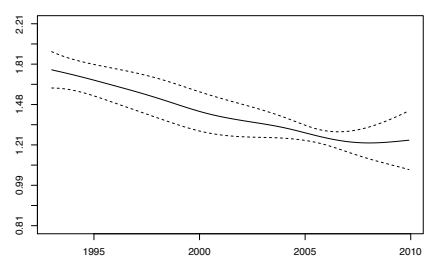

(b) age $35-49$

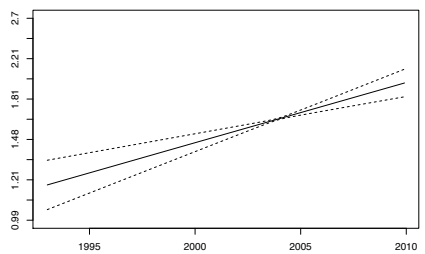

(e) high school

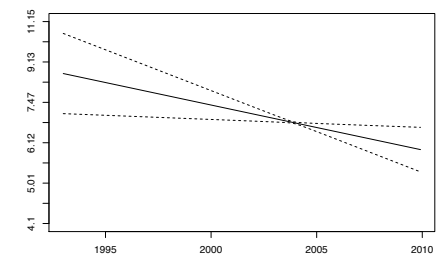

(h) low income

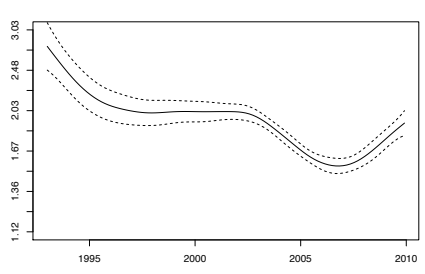

(j) does not work

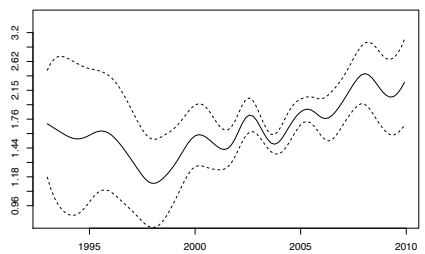

(1) Hispanic

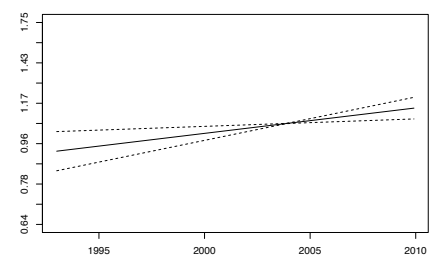

(o) two health conditions

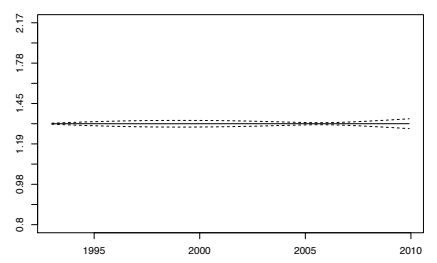

(c) male

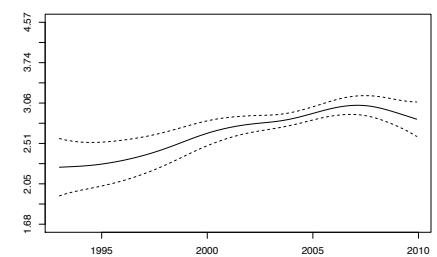

(f) grade 11 or less

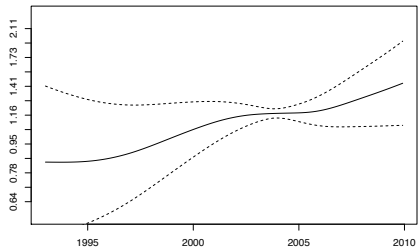

(m) black

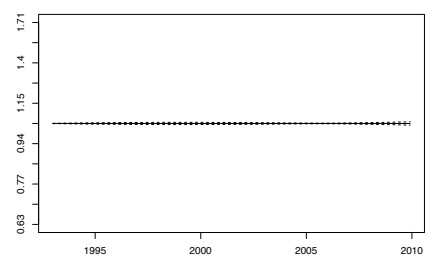

(p) three health conditions

Figure 1: Odds ratio plots of time varying coefficient model for having no health plan. 
represent lifetime effects of socioeconomic limitations, and may influence future occupational opportunities and earning potential. Higher educational degrees are typical prerequisites for highly compensated work, which allows affordability of health insurance coverage and access to high-quality care (Muller, 2002).

Similar to the finding of the previous literature, our results show that non-Hispanic whites are more likely than any other race/group to have insurance coverage (Ahluwalia \& Bolen, 2008; Cohen \& Martinez, 2013). The results from the varying coefficient model showed that there is an increased odds ratio trend (although less clearly for the Hispanic category) for not having a health plan for African Americans and Hispanics compared with the white race reference group, indicating, therefore, the disparities are increasing. The racial disparities in health insurance are strongly associated with socioeconomic factors: African Americans and Hispanics face greater economic and educational barriers than other groups and have less access to high-paying jobs that facilitate access to health care and health care coverage. Compared with non-Hispanic whites, non-Hispanic blacks and Hispanics tend to have higher rates of unemployment and under-representation in good-paying jobs that include health insurance as part of the benefit package (Muller, 2002). Furthermore, individuals from racially or ethnically diverse backgrounds make up a great majority of the uninsured population (Cohen \& Martinez, 2013). Since the nation's population continues to become increasingly diverse (people of color are projected to comprise more than $50 \%$ of the U.S. population by 2050), this disparity in insurance coverage is likely to grow if left unaddressed (Andrulis et al., 2013).

The results fit in a rapidly changing scenario that we briefly present to better understand how the results of our analysis is influenced or will be influenced by recent and future decisions. Health insurance coverage has long been regarded as a facilitator to health care access; eliminating disparities in health insurance coverage is certainly one necessary component to reducing disparities in health outcomes, especially for the most vulnerable groups. In the US, there have been several healthcare reform proposals with the aim of improving health care coverage, and consequently health care access to address this vulnerability: the most ambitious of recent legislation, the Affordable Care Act, became law in March of 2010. As is implied in the title of the ACA, one of the key provisions in the law is the expansion of coverage to the socially and economically disadvantaged population and making health care more affordable.

It is still too early to evaluate the ability of ACA to reduce disparity in health insurance and health care access. Some authors have pointed out some elements of weakness in the ACA (McDonough, 2014; McDonough \& Adashi, 2014; Molinari, 2014). A major concern with respect to the expansion of Medicaid is that participation of health care providers in Medicaid is in jeopardy: the huge increase in the number of individuals covered by state insurance through raising the threshold of those families and individu- 
als eligible for Medicaid, may create an increase in the number of requests for healthcare at tariffs fixed by the individual States as happens within the Medicaid program. Already now, however, only a limited number of doctors are ready to offer their services for reimbursement tariffs that have been leveled down by the Medicaid program (Moy et al., 2011; Decker, 2012). A potential risk is that, seeing that there will not be a corresponding increase in services, this massive rise in numbers of people insured by Medicaid may increase the average waiting time for Medicaid covered patients leading to lower access to health care.

Moreover, the extension of Medicaid and the subsidies available to economically disadvantaged individuals may not be utilized if the low income individuals and families are not aware of these options. Individuals with low education not only have difficulty reading printed health materials, they also struggle to understand technical terms, jargon, and complex concepts that are often embedded in Medicaid insurance program (Rudd et al., 2005). Therefore, states should be more aggressive in facilitating outreach efforts to effectively reach health care consumers and educate them about the ACA, ensuring that materials such as enrollment and claim information and financial disclosures are in plain language. Also from our results education appears per se as one of the drivers to address in order to reduce the gaps.

Finally, under the ACA, insurance companies will be obliged to include in the insurance plan some "essential" services, such as mental health treatment and some preventative measures. Of course, compulsory inclusion of this type of care into the coverage is an important innovation that aims at guaranteeing a level of insurance provision that is in line with the services provided in many European health care systems, producing, eventually, a decrease in health disparities. However, the apparent "guarantee" of more extensive coverage, has meant initially several cancellations by the insurance companies who have been informing many of their insured that it will be impossible for them to renew their policy because it is not in line with some of the "essential" characteristics laid down by the law. If these "side effects" are not taken into account this could result in a potential increase of the disparities in health care coverage, in particular for those who do not receive health insurance either through the state or their employer having to buy insurance coverage that indeed will comply legally but for sure will be more expensive.

The challenge still remains in "closing the gap" and inverting the negative trends we have clearly shown in our analysis. Much is left to accomplish in order to prevent and reduce barriers that make it difficult to obtain basic health care coverage and medical care, either through the implementation of the ACA or other interventions. In the era of big reforms addressing these issues, surveillance programs such as BRFSS can provide useful data for monitoring and evaluating their effectiveness. 


\section{Conclusion}

Our study indicates that the disparities as well as temporal trends exist in many of the socioeconomic factors that are associated with health care access. The use of the varying coefficient model method was able to show how these disparities are changing over time and whether the gaps in the access to health care coverage have been improving or not. This provides significantly more information than observing changes in proportions between two periods of time for instance. The trends can be used to inform health policy interventions and understand which subgroups of the population may require specific attention. Future work should focus on the impact of the other factors such as geographic variation and the interaction with the socioeconomic status on healthcare access, and how the effect of ACA is changing the disparities and temporal trends of these factors. 


\section{References}

Ahluwalia, I. B., \& Bolen, J. 2008. Lack of health insurance coverage among working age adults, evidence from the Behavioral Risk Factor Surveillance System, 1993 to 2006. Journal of Community Health, 33(5), 293-296.

AHRQ. 2007. Questions and answers about health insurance: a consumer guide. Tech. rept. 07-0043. Agency for Healthcare Research Quality (AHRQ), America's Health Insurance Plans.

Andrulis, D. P., Siddiqui, N. J., Cooper, M. R., \& Jahnke, L. R. 2013. The affordable care act and racial and ethnic health equity series: report No. 3 enhancing and diversifying the nation's health care workforce. Tech. rept. Texas Health Institute. http://phetoolkit.net/docs/aca_equity_workforce_report_09.13.2013_final.pdf.

CDC. 2012. Methodologic Changes in the Behavioral Risk Factor Surveillance System in 2011 and Potential Effects on Prevalence Estimates. Center for Disease Control and Prevention. Available at: http://www.cdc.gov/mmwr/preview/mmwrhtml/mm6122a3.htm. Retrieved on: 19 May 2014.

CDC. 2014. About the Behavioral Risk Factor Surveillance System (BRFSS). Center for Disease Control and Prevention. Available at: http://www.cdc.gov/brfss/about/about_brfss.htm. Retrieved on: 5 January 2014.

Cohen, R. A., \& Martinez, M. E. 2013. Health insurance coverage: early release of estimates from the National Health Interview Survey, January-March 2013. Tech. rept. Center of Disease Control and Prevention.

Cohen, Robin A, \& Bloom, Barbara. 2010. Access to and utilization of medical care for young adults ages 20-29 years: United States, 2008. NCHS data brief, 1-8.

Connors, E. E., \& Gostin, L. O. 2010. Health care reform - a historic moment in US social policy. Journal of the American Medical Association, 303(24), 2521-2522.

DECKER, S. L. 2012. In 2011 nearly one third of physicians said they would not accept new Medicaid patients, but rising fees may help. Health Affairs, 31(8), 1673-1679.

Eilers, P. H. C., \& Marx, B. D. 1996. Flexible smoothing with B-splines and penalties. Statistical Science, 89-102.

Eilers, P. H. C., \& Marx, B. D. 2002. Generalized linear additive smooth structures. Journal of Computational and Graphical Statistics, 11(4), 758-783. 
FAN, J., \& ZhANG, W. 2008. Statistical methods with varying coefficient models. Statistics and Its Interface, 1(1), 179.

Hastie, T., \& Tibshirani, R. 1993. Varying-coefficient models. Journal of the Royal Statistical Society. Series B (Methodological), 757-796.

Hoover, D. R., Rice, J. A., Wu, C. O., \& Yang, L. P. 1998. Nonparametric smoothing estimates of time-varying coefficient models with longitudinal data. Biometrika, 85(4), 809-822.

Huang, J. Z., Wu, C. O., \& Zhou, L. 2002. Varying-coefficient models and basis function approximations for the analysis of repeated measurements. Biometrika, 89(1), 111-128.

Huang, J. Z., Wu, C. O., \& Zhou, L. 2004. Polynomial spline estimation and inference for varying coefficient models with longitudinal data. Statistica Sinica, 14(3), 763-788.

Light, D. W. 2011. Historical and comparative reflections on the US national health insurance reforms. Social Science \& Medicine, 72(2), 129-132.

Marx, B. D. 2010. P-spline varying coefficient models for complex data. Statistical Modelling and Regression Structures, 19-43.

McDonough, J. E. 2014. Health system reform in the United States. International Journal of Health Policy and Management, 2, 5-8.

McDonough, John E, \& Adashi, Eli Y. 2014. Realizing the Promise of the Affordable Care Act—January 1, 2014. JAMA, 311(6), 569-570.

Medicare. 2014. About the Medicare Part D (Prescription Drug) Donut Hole, or Coverage Gap. Available at: http://medicare.com/medicarepartd/about/whatisthedoughnuthole. Accessed on: 17 June 2014.

Meyer, P. A., Yoon, P. W., Kaufmann, R. B., Office for State, Tribal, \& THE CDC. 2013. CDC health disparities and inequalities report - United States 2013. Morbidity and Mortality Weekly Report - Center for Disease Control and Prevention, 62(SU-3), 3-5.

MokdAD, A. H. 2009. The behavioral risk factors surveillance system: past, present, and future. Annual review of public health, 30, 43-54.

Molinari, Carol. 2014. Does the Accountable Care Act aim to promote quality, health, and control costs or has it missed the mark? Comment on "Health system 
reform in the United States". International journal of health policy and management, $2(2), 97$.

Moy, B., Polite, B. N., Halpern, M. T., Stranne, S. K., Winer, E. P., Wollins, D. S., \& Newman, L. A. 2011. American Society of Clinical Oncology policy statement: Opportunities in the Patient Protection and Affordable Care Act to reduce cancer care disparities. Journal of Clinical Oncology, 29(28), 3816-3824.

MulleR, A. 2002. Education, income inequality, and mortality: a multiple regression analysis. British Medical Journal, 324(7328), 23.

National Center for Health Statistics. 2013. Health, United States, 2013: With Special Feature on Prescription Drugs. Center for Disease Control and Prevention. Available at: http://www.cdc.gov/nchs/data/hus/hus13.pdf. Retrieved on: 19 May 2014.

Rowland, D., \& Lyons, B. 1996. Medicare, Medicaid, and the elderly poor. Health Care Financing Review, 18(2), 61-85.

Rudd, R. E., Renzulli, D., Pereira, A., \& Daltroy, L. 2005. Understanding health literacy: implications for medicine and public health. American Medical Association Press. Chap. Literacy demands in health care settings: the patient perspective, pages $69-84$.

Schoen, C., Osborn, R., Squires, D., \& Doty, M. M. 2013. Access, Affordability, And Insurance Complexity Are Often Worse In The United States Compared To Ten Other Countries. Health Affairs, 32(12), 2205-2215.

Strine, T. W., Zack, M., Dhingra, S., Druss, B., \& Simoes, E. 2011. Uninsurance among nonelderly adults with and without frequent mental and physical distress in the United States. Psychiatric Services, 62(10), 1131-1137.

Sudano, J. J., \& Baker, D. W. 2006. Explaining US racial/ethnic disparities in health declines and mortality in late middle age: the roles of socioeconomic status, health behaviors, and health insurance. Social Science $\&$ Medicine, 62(4), 909-922.

United Health Foundation. 2013. America's Health Rankings: A Call to Action for Individuals and Their Communities. Available at: http://www.americashealthrankings.org/reports/annual. Retrieved on: 19 May 2014.

U.S. Bureau OF LABOR Statistics. 2014. The employment situation - February 2014. Tech. rept. Bureau of Labor Statistics. http://www.bls.gov/news.release/pdf/empsit.pdf. 
US Department of Health and Human Services. 2013. Prior HHS poverty guidelines. Available at: aspe.hhs.gov/poverty/figures-fed-reg.cfm. Accessed on: 5 August 2013.

William, D. R., \& Collins, C. 1995. US socioeconomic and racial differences in health: patterns and explanations. Annual Review of Sociology, 21, 349-386.

Williams, D. R., \& Collins, C. 2001. Racial residential segregation: a fundamental cause of racial disparities in health. Public Health Reports, 116(5), 404.

Wood, S. N. 2006. Generalized additive models: an introduction with R. Vol. 66. Chapman \& Hall. 